\title{
Motivational Interviewing and Specialty Pharmacy
}

\author{
Bruce A. Berger, PhD, and Carl T. Bertram, PharmD
}

\begin{abstract}
SUMMARY
It is well documented in substance abuse and health care literature that motivational interviewing is an evidenced-based and effective intervention for influencing patient behaviors and associated positive health outcomes. The introduction of motivational interviewing training in specialty pharmacy has great potential to increase patient and pharmacist satisfaction, maximize adherence rates, and improve health outcomes.

This commentary examines the need for effective approaches for improving patient adherence and outcomes and briefly describes the history and efficacy of motivational interviewing. Case studies using traditional approaches to patient care and motivational interviewing are analysed, and real-world experience using motivational interviewing is presented in the form of a specialty pharmacy case study.
\end{abstract}

J Manag Care Pharm. 2015;21(1):13-17

Copyright @ 2015, Academy of Managed Care Pharmacy. All rights reserved.

I dentifying new interventions for managing specialty pharmacy trends and associated health care costs are important now more than ever with the inflation of specialty pharmaceuticals, including associated new products entering the market. Payers of specialty pharmaceuticals are searching for new ways of ensuring that the value of their dollar is optimized with patients requiring these medications. Once it is determined that the high-cost specialty product is clinically appropriate and the medication is dispensed, it is crucial to ensure that the patient is self-motivated and appropriately educated to use the medication according to the physician's instructions. Enter motivational interviewing as an efficacious means to improve adherence to appropriately prescribed specialty pharmaceuticals. ${ }^{1}$

Motivational interviewing (MI) was originally developed by William Miller and Stephen Rollnick. Miller's insightful reaction to the confrontational approach widely used in the field of addiction counseling in the 1980s was the start of MI. In this confrontational approach, substance abusers were seen as "pathological liars with formidable immature personality defenses, in denial and out of touch with reality." "Counselors were taught to confront this denial in order to establish what these substance abusers had to do to re-engage reality and to return to health. However, Miller experienced something different with these patients:

Because I knew very little about alcoholism I relied heavily on listening to clients on the ward, learning from them and trying to understand their dilemma. I found them usually to be open, interesting, thoughtful people well aware of the chaos ensuing from their drinking. That's why, when I began reading clinical descriptions, I thought, 'That doesn't sound at all like the same people I've been seeing!'2

Miller eventually came to the fundamental realization that the patient's openness to change is directly affected by how the counselor talks to the patient. When people are treated with contempt or disrespect, change is unlikely. When they are treated with care, concern, and respect, change is possible. ${ }^{1}$

Counsel in a way that evokes defensiveness and counter-argument and people are less likely to change. ... I set out, then, to discover how to counsel in a way that evokes people's own motivation for change rather than putting them on the defensive. A simple principle that emerged from our earliest discussions was to have the client, not the counselor, voice the reasons for change. ${ }^{2}$

There is evidence of the efficacy of MI in substance abuse, ${ }^{3}$ and it might prove to be an effective way of intervening for other health behavior issues.

MI is a patient-centered form of counseling that helps patients to reason their way to the conclusion that they need to change their behaviors in order to achieve their goals. It is founded on Carl Rogers' client-centered counseling. Rogers emphasized that the relationship with the patient had to be based on unconditional positive regard for the patient as a person and therefore avoids shaming and blaming the patient. Only by valuing and supporting the patient as a person does the patient have the freedom and safety to examine his or her behaviors and their consequences for others. ${ }^{1}$

While embracing the relationship advocated by Rogers, Miller modified Rogers' nondirective method for how to talk with the patient. Rogers believed that if the counselor accurately and consistently reflected back to the patient what the patient was saying, the patient would eventually achieve selfinsight and start to see the need to change. ${ }^{4}$ Although this Rogerian approach to counseling was effective, it can be slow and time consuming. Miller sought a more directive or guiding way of addressing the patient that does not cause defensiveness in the patient and helps the patient to conclude that change is both needed and possible. The counselor provides care, respect, and insight or information to guide the patient to new or healthier options. The patient is invited to draw new conclusions about goals and behaviors. The counselor is thus a caring and respectful resource for the patient. ${ }^{1}$ 
MI does 2 important things: (1) it accurately and nonjudgmentally reflects the concerns and emotions of the patient, and (2) it provides insight or new information to address the concerns in a nonjudgmental and nonthreatening manner. This combination is powerful. It creates safety so that the patient can be open to new ideas, learn, draw new conclusions, and, consequently, engage in new behaviors. It should be clear that MI was developed to address ambivalence and/or resistance to change. Clearly, if a patient is ready to do something (e.g., quit smoking, lose weight, or take a medication), then telling the patient what to do might be useful. However, when the patient is ambivalent, this telling or persuasive approach actually produces the opposite effect. That is, when people are not ready to change, telling them what to do actually forces them to defend the very behavior we are attempting to influence. The patient digs in and presents arguments to counter the proposed change.

\section{Evidence of How MI Works in Health Care}

Various studies demonstrate the effectiveness of MI in increasing patient and provider satisfaction and producing better outcomes. One such study, Berger et al. (2005), was conducted for Biogen Idec concerning the specialty drug Avonex for multiple sclerosis. ${ }^{5}$ In a randomized controlled clinical trial, patients in the MI intervention group demonstrated a statistically significantly lower proportion of Avonex treatment (1.2\%) discontinuation than the standard care group ( $8.7 \%)$. In addition, movement toward continuation of therapy was significantly higher in the treatment group. Given that Avonex was a weekly injection that at the time of the study cost approximately $\$ 200$ a dose, 9,000 less dropouts represented a $\$ 93,600,000$ cost recovery per year $(9,000$ patients $\times \$ 200 /$ dose $\times 52$ weeks/ year $=\$ 93,600,000) .{ }^{5}$ These positive results illustrate our belief that MI can bring about major health behavior change by patients.

In one of the most comprehensive reviews of the effectiveness of MI in health care settings, Rubak et al. (2005) did a systematic review and meta-analysis of MI. ${ }^{6}$ The authors reviewed 72 randomized control clinical trials using MI. Meta-analysis showed a significant effect (95\% confidence interval) for MI for body mass index, total blood cholesterol, systolic blood pressure, and blood alcohol concentration and standard ethanol content. MI had a significant and clinically relevant effect in approximately 3 out of 4 studies, with an equal effect on physiological (72\%) and psychological (75\%) diseases. Psychologists and physicians obtained an effect in approximately $80 \%$ of the studies, while other health care providers obtained an effect in $46 \%$ of the studies. When using MI in brief encounters of 15 minutes, 64\% of the studies showed an effect. More than 1 encounter with the patient seemed to ensure the effectiveness of MI. The authors concluded that MI outperforms traditional advice giving in the treatment of a broad range of behavioral problems and diseases. ${ }^{6}$
In specialty pharmacy, specifically, MI has been shown to be statistically and clinically significant in treating HIV (Mansoor et al., 20147), rheumatoid arthritis (Rau et al., 2008 ), and chronic obstructive pulmonary disease (Tolijamo et al., 2010 $)$.

\section{A New Approach to MI}

As stated previously, MI was originally conceived to help patients with addictions. There are 3 fundamental ways in which teaching MI in health care is different from teaching MI as originally conceptualized for substance abuse: (1) the training of health care professionals (HCPs), (2) the patient or client, and (3) the context of treatment.

First, the context of the relationship between HCPs and their patients is often different from the relationship between counselors/psychologists and their clients. ${ }^{1}$ Next, how counselors are trained is different from the training of HCPs. Counselors are taught to explore the patients' problems and that patients must eventually draw their own conclusions if change is to occur. Counselors see their role as being supportive and caring and providing insight. Patient are "in charge" because it is ultimately their lives and therefore their decisions.

HCPs are taught that they are the experts and they are in charge. This orientation creates many problems for HCPs when attempting to adopt patient-centered approaches. HCPs have to learn an entirely different way of talking, which counselors have already learned before they are exposed to MI. When learning MI, HCPs must learn that they are not in control and that they are not the only expert in the room. Patients are experts, too. They are experts on their lives, their goals, and their aspirations. Equally important, they are experts on their "sense making." How do patients make sense out of what is happening to them? What is their understanding of diabetes, high cholesterol, hypertension, etc.? What do they understand may happen if these illnesses are not treated? How does the way patients make sense of their illnesses and treatments affect their motivation for change and their emotional responses to the diagnosis and treatment? Generally speaking, patients will always develop their own theories and reasoning about all of these things. We need to understand what the patient knows, understands, and believes before our expertise can be useful. And, we must respectfully acknowledge these things first. Without understanding how patients construct their ideas about illness and risk, HCPs really cannot know what information or education might be useful or meaningful to the patients.

Unfortunately, the medical model (implemented as a clinical workup) is often mechanical and formulaic and does not thoroughly take into account the patient's perspective. In fact, the clinical workup is linear-do this, then this, and finally this. Patients, however, are not linear. They may not be ready to "be worked up." In fact, it is folly to skip to the workup without first finding out how patients are making sense out of what is happening to them. It is their story not ours. In many ways, MI 
must undo and replace the medical model that puts the clinician as the sole expert and "in control." This is no easy task. HCPs have been used to giving directions, telling patients what to do, and then blaming patients when there is "failure." The approach taken by MI is one of guiding patients, collaborating, and not dictating to them. MI is about providing patients with options that fit the patient's larger goals. How does managing asthma or diabetes or cholesterol align with the broader aspirations of the patient?

Another important distinction between the training of HCPs and the training of counseling and clinical psychologists is the issue of introspection. Counseling and clinical psychologists are trained to be introspective and conscious of how their own needs or "issues" could affect or contaminate the relationship. Through practicums and mentoring during their training, they are made aware of these needs so that they do not interfere with their client relationships.

Interestingly, despite the fact that HCPs work directly with patients concerning their health and make critical decisions about their health, the curricula for many HCPs does not in any appreciable way broach the subject of introspection, consciousness, and transference and countertransference. Current curricula do focus on reflective practice; however, the focus is often on decision making, knowledge, and skills. Greater emphasis is needed on introspective reflection by HCPs to assess how one's self-concept affects interactions with patients. Moreover, personal reflection of this nature must be guided and mentored by someone experienced in identifying and resolving these issues in practitioners and students. HCP training must address this subject. It is simply too important to ignore, especially when learning MI.

A simple example illustrates this point. ${ }^{1}$ After intensive training in MI, a nurse expressed her frustration with many of her patients. She said, "I'm a doer. I get things done. Many of them just don't want to move forward. I find myself getting frustrated with them and I literally forget what I have learned about MI." The nurse was asked the following questions: "When your patients don't move forward as fast as you would like, what rewards that you get as a doer must be suspended? How does that affect how you see yourself and your success with the patient? In other words, can you see a time when you will be able to bear not defining your success through your patients' decisions? Can you suspend being a doer (or focus doing on MI)?" These questions had a profound impact on this nurse, and she began to see how her definition of success and self were actually undermining her ability to be fully present (caring, nonjudgmental, and patient). So, in addition to having to learn and master the skills and spirit of MI (which really fly in the face of the standard medical expert model), HCPs also must learn to become introspective and aware of how their personal assumptions and issues can actually interfere with the ability to influence patient decision making in a positive and nonthreatening way. This makes learning and mastering MI even more difficult for HCPs.

In addition to issues surrounding HCP training, patients with chronic illnesses are different from clients struggling with substance abuse or marital discord. Traditional MI assumes that patients have everything they need internally to make changes. The role of the "counselor" is to help the patient discover and activate those resources and come to better conclusions about their behaviors. We agree that at some level patients with personal or substance abuse problems often know all the pros and cons associated with their behavior. However, while patients managing chronic illness have the same set of internal psychological resources, they often do not have everything they really need to manage an illness, such as diabetes. For example, they often have misconceptions about the illness and its severity, especially if left untreated. This is particularly true if the illness is asymptomatic. Sometimes they really do not see the point in treating the illness because they "feel fine." A well-trained HCP, using MI, can assist the patient in making healthier decisions by filling in gaps in the patient's understanding or knowledge and by then inviting the patient to respond to this information. A well-trained HCP using MI is aware that without a strong foundation of rapport with the patient, information can be interpreted by the patient as a way of putting them in their place or correcting them, rather than as an extension of the care provided by the health professional.

Finally, HCPs often use MI in a different treatment context than substance abuse counselors because HCPs often do not have the luxury of repeated 50- to 60-minute encounters. In fact, many times the HCP may have only 1 chance to make an impact on a patient because continuity of care is not where it needs to be in health care. Therefore, the approach taken here leans toward brief MI in the form of 5- to 30-minute encounters.

\section{Using MI in Health Care: An Example}

One of the best ways to illustrate how MI works compared with a traditional health care encounter is through an example. In the following example, the traditional response is presented first, followed by the MI response.

Here is the case: Emily Franklin has multiple sclerosis (MS). She was prescribed a once-a-week intramuscular injection to treat her MS. She is displeased with how her medication is working.

\section{Traditional Response}

Emily: I don't understand why I should keep using this medication. It doesn't work. I had another flare-up last week.

HCP: Just because you had a flare-up doesn't mean it isn't working.

Emily: That doesn't make sense. Why take it if it isn't going to cure my MS?

HCP: It doesn't cure MS. It slows the progression of your 
illness. You will still have flare-ups, but they probably won't occur as often or be as strong.

Emily: I don't know that I want to keep using it if it doesn't cure my MS.

HCP: Nothing will cure your MS ... only slow it down. It will slow the progression, as I already said.

Emily: Then I don't see the point.

HCP: Okay... you do want you want. It's your life.

Discussion. What happened here? Why is Emily not listening to what her HCP is telling her? Primarily, it is because she does not feel understood. Rather than acknowledging Emily's frustration, the HCP constantly corrects her. Consequently, Emily feels lectured to and that her concerns are not respected. Her sense is that the medication should cure her MS, and she is surprised and disappointed when she has another flare-up. None of this is acknowledged by the HCP. As a result, even though the HCP gives Emily factual information, she does not accept it. Let's look at how an HCP trained in MI might handle the situation.

\section{Motivational Interviewing Response}

Emily: I don't understand why I should keep using this medication. It doesn't work. I had another flare-up last week.

HCP: Because you had another flare-up of your MS, you have serious doubts about whether the medication is still working. Emily: Exactly... why stick myself with a needle when it doesn't help?

HCP: You raise a good question. It doesn't make sense to give yourself an injection of medication that doesn't seem to work. Would you mind if I shared some thoughts with you about the medicine and you tell me what you think?

Emily: Okay. I guess.

HCP: While there is currently no cure for MS, this medication is very effective in slowing down the progression of the illness. You may still have a flare-up, but they will occur less often and generally be less powerful than before. I wish there was a cure. I would hate to see your MS progress more rapidly without the medication. What do you think about this new information?

Emily: Well, I thought it cured my MS.

HCP: And, that's why you were surprised when you had a flare-up.

Emily: Right.

HCP: I know that you're disappointed. I would worry if you stopped using the medication because your MS could progress at a much faster rate without it. I would hate to see that happen. Where does this leave you now in regard to continuing to use the medication?

Emily: I don't want my MS to progress faster. I'll keep using the medication.
HCP: I am glad that you are committed to continuing to use the medication.

Discussion. In this conversation, the HCP acknowledges the surprise, frustration, and disappointment of the patient. He listens to her concerns and accurately reflects back how she is making sense of the situation. He then asks permission to share new information that invites her to consider continuing to use the medication. After providing new information about how the drug works, he asks Emily where this leaves her now in regard to continuing to use the medication. He tells her that he is concerned about her MS progressing. He overtly lets her know that he cares about her and her health. In the end, she chooses to continue to use the medication. Notice that the HCP does not make any decisions for the patient or draw conclusions for her.

\section{Future of MI in Pharmacy Practice}

We have observed that schools of pharmacy do a great job of preparing students to know drug therapy. Opportunity exists, however, for better preparation to know people and how to address problems of behavior change (e.g., taking medications, losing weight, or quitting smoking).

Current clinical outreach processes in pharmacy benefit management companies and the specialty pharmacy industry have focused on traditional means of communication such as telephone, interactive voice response, and fax. The message from these communications have focused on "recommending/ telling" patients and providers what to do based on national guidelines or what their insurance companies want (e.g., what the benefit design is). There may be less focus on understanding patients' abilities to make sense of their current conditions or current medication therapies (patient-centered approach). This must be the starting point.

Building a culture of communicating with patients and providers at the right moments of opportunity, through patientcentered MI practices (e.g., phone and video consults), is a method that can be used to optimize patient outcomes.

\section{Conclusions}

Being patient centered, that is, knowing how to demonstrate a caring attitude toward patients, the ability to help patients make sense of their conditions or their medications, and having excellent listening skills are essential for pharmacists (and other ancillary health care providers) to influence positive health behaviors. Ultimately, this will result in better health outcomes for patients and reduced expenditures on health care. 


\section{Authors}

BRUCE A. BERGER, PhD, is President, Berger Consulting, LLC, and Professor Emeritus, Auburn University, Auburn, Alabama, and CARL T. BERTRAM, PharmD, is Chief, Specialty Pharmacy Clinical Strategy, Cigna, Bloomfield, Connecticut, and former Vice President, Innovation/Clinical Strategy, Catamaran/Briova, Chicago, Illinois.

AUTHOR CORRESPONDENCE: Bruce A. Berger, PhD;

Tel.: 334.444.3160; E-mail: Bbergerconsulting@gmail.com.

\section{DISCLOSURES}

The authors declare no conflicts of interest, financial or otherwise.

Concept and design and manuscript writing and revision were primarily contributed by Berger, along with Bertram. Both authors contributed equally to data collection and interpretation.

\section{REFERENCES}

1. Berger BA, Villaume WA. Motivational Interviewing for Health Care Professionals: A Sensible Approach. Washington, DC: American Pharmacists Association Press; 2013.
2. Miller WR, Rollnick S. Motivational Interviewing in Health Care: Helping People Change. Applications of Motivational Interviewing Series. 3rd ed. New York: Guilford Press; 2012.

3. Kazemi DM, Levine MJ, Dmochowski J, Nies MA, Sun L. Effects of motivational interviewing intervention on blackouts among college freshmen. J Nurs Scholarsh. 2013;45(3):221-29.

4. Rogers Carl R. On Becoming a Person. New York: Mariner Books; 1961.

5. Berger BA, Liang H, Hudmon KS. Evaluation of software-based telephone counseling to enhance medication persistency among patients with multiple sclerosis. J Am Pharm Assoc (2003). 2005;45(4):466-72.

6. Rubak S, Sandbæk A, Lauritzen T, Christensen B. Motivational interviewing a systematic review and meta-analysis. Br J Gen Pract. 2005;55(513):305-12.

7. Mansoor LE, Karim QA, Werner L, et al. Impact of an adherence intervention on the effectiveness of tenofovir gel in the CAPRISA 004 trial. Aids Behav. 2014;18(5):841-48.

8. Rau J, Ehlebracht-König I, Petermann F. [Impact of a motivational intervention on coping with chronic pain: results of a controlled efficacy study]. Schmerz. 2008;22(5):575-78, 580-85. [Article in German].

9. Toljamo T, Kaukonen M, Nieminen P, Kinnula VL. Early detection of COPD combined with individualized counselling for smoking cessation: a two-year prospective study. Scand J Prim Health Care. 2010;28(1):41-46. 